\title{
From nonalcoholic fatty liver disease to metabolic dysfunction-associated fatty liver disease: is it time for a change of terminology?
}

\author{
Giovanni Targher ${ }^{1}$, Christopher D. Byrne ${ }^{2}$ \\ 'Department of Medicine, Section of Endocrinology, Diabetes and Metabolism, University of Verona, Verona 37126, Italy. \\ ${ }^{2}$ Southampton National Institute for Health Research Biomedical Research Centre, University Hospital Southampton, \\ Southampton General Hospital, Southampton SO16 6YD, United Kingdom.
}

Correspondence to: Prof. Giovanni Targher, Department of Medicine, Section of Endocrinology, Diabetes and Metabolism, University of Verona, Verona, Piazzale A. Stefani 1, Verona 37126, Italy. E-mail: giovanni.targher@univr.it

How to cite this article: Targher G, Byrne CD. From nonalcoholic fatty liver disease to metabolic dysfunction-associated fatty liver disease: is it time for a change of terminology? Hepatoma Res2020;6:64. http://dx.doi.org/10.20517/2394-5079.2020.71

Received: 27 Jul 2020 First Decision: 18 Aug 2020 Revised: 19 Aug 2020 Accepted: 19 Aug 2020 Published: 12 Sep 2020

Academic Editor: Stefano Bellentani Copy Editor: Cai-Hong Wang Production Editor: Jing Yu

\begin{abstract}
Nonalcoholic fatty liver disease (NAFLD) has become the most common cause of liver disease in many parts of the world, causing considerable liver-related (steatohepatitis, cirrhosis, liver failure and hepatocellular carcinoma) and extra-hepatic morbidity and mortality (mainly cardiovascular disease, chronic kidney disease or certain types of extra-hepatic cancers). Recently, based on insights gained from the past two decades, an international panel of experts from 22 countries has taken the initiative to propose a new name and definition for NAFLD in adult individuals - that is, metabolic dysfunction-associated fatty liver disease. This proposed change in nomenclature is not simply a semantic revision, but may facilitate improved diagnosis of this common liver disease for health promotion, case identification, patient awareness, ongoing clinical trials and health services delivery. The aim of this commentary is to discuss the proposal for a change in nomenclature of this common and burdensome liver disease and to address the "pros and cons" for changing the name according to the perspective of different stakeholders.
\end{abstract}

Keywords: Nonalcoholic fatty liver disease, metabolic dysfunction-associated fatty liver disease, liver fat, commentary 


\section{MAIN TEXT}

Nonalcoholic fatty liver disease (NAFLD) is a growing global health problem that affects about a quarter of the world's adult population and poses a major health and economic burden to all societies ${ }^{[1-3]}$. Recently, two new position articles, published by a panel of international experts from 22 countries, have proposed a change of terminology and definition for NAFLD, called "metabolic dysfunction-associated fatty liver disease" (MAFLD) ${ }^{[4,5]}$.

As summarized in the schematic Figure 1, over the last two decades there have been concerns expressed by both individual experts and some special conferences of scientific societies (an AASLD 2003 single topic conference and an EASL 2009 special conference, respectively) regarding the inaccuracy and possible "negative" consequences of using the term "NAFLD" to describe a fatty liver disease associated with metabolic dysfunction ${ }^{[6-13]}$. Since the initial descriptions in the early 1980 s by Ludwig et al. ${ }^{[6]}$, Schaffner and Thaler ${ }^{[7]}$, who coined the terms of nonalcoholic steatohepatitis (NASH) and NAFLD, respectively, to describe a fatty liver disease arising in the absence of significant alcohol consumption, there have been major conceptual advances in our understanding of the complex pathophysiological mechanisms of this common liver disease.

Although the change of nomenclature from NAFLD to MAFLD that has been recently proposed by the panel of international experts is still under discussion, it is important to underline that this change in terminology is not merely a semantic revision, but can also represent the first step toward a better identification of this common and burdensome metabolic liver disease for improved health promotion, case identification, patient awareness, ongoing clinical trials and health services delivery ${ }^{[5,6,14-16]}$.

\section{Criteria for MAFLD diagnosis}

The current definition of NAFLD is based on the presence of hepatic steatosis (detected by liver biopsy, imaging methods or blood biomarkers/scores) in the absence of significant alcohol consumption (though the currently recommended cut-offs to define "significant" alcohol consumption are arbitrary) and the exclusion of other secondary causes of hepatic steatosis ${ }^{[17-19]}$. Interestingly, the newly proposed definition of MAFLD shifts from a liver disease of "exclusion" (i.e., non-alcoholic fatty liver without coexisting known causes of fatty liver) to one of "inclusion", as the newly proposed diagnostic criteria are based on the presence of hepatic steatosis, in addition to one of the following three criteria (namely overweight/obesity, presence of established type 2 diabetes mellitus, or evidence of metabolic dysregulation), regardless of daily alcohol consumption and other concomitant liver diseases ${ }^{[5]}$. A flowchart for the proposed simple criteria for the diagnosis of MAFLD in adult individuals is depicted in Figure 2. It is important to underline that these diagnostic criteria do not apply to paediatric population ( $<18$ years), because different cut-off points for defining the presence of overweight/obesity and other metabolic risk abnormalities should be used in children and adolescents. As also shown in this figure, the criteria proposed for diagnosing the presence of metabolic dysregulation among lean/normal weight individuals with hepatic steatosis who do not have type 2 diabetes, are the presence of at least two metabolic risk factors from: (1) those risk factors that are widely used to identify the metabolic syndrome (using ethnic- and country-specific cutoff points of increased waist circumference $)^{[20]}$; (2) a homeostasis model assessment-estimated insulin resistance score $\geq 2.5$; or (3) a plasma high sensitivity-C-reactive protein (hs-CRP) level $>2 \mathrm{mg} / \mathrm{L}$. It is well known that the metabolic syndrome is a complex of inter-related risk factors for both type 2 diabetes and cardiovascular disease. These risk factors include dysglycaemia, hypertension (or raised blood pressure), atherogenic dyslipidemia (typically defined by increased plasma triglycerides and low high-density lipoprotein cholesterol) and obesity (particularly increased abdominal adiposity) ${ }^{[20]}$. Interestingly, the new definition of MAFLD also includes elevated plasma hs-CRP levels as one of the metabolic risk factors, because it is well established that this plasma inflammatory biomarker (mostly secreted by the liver) is also often increased with cardiometabolic disorders. 


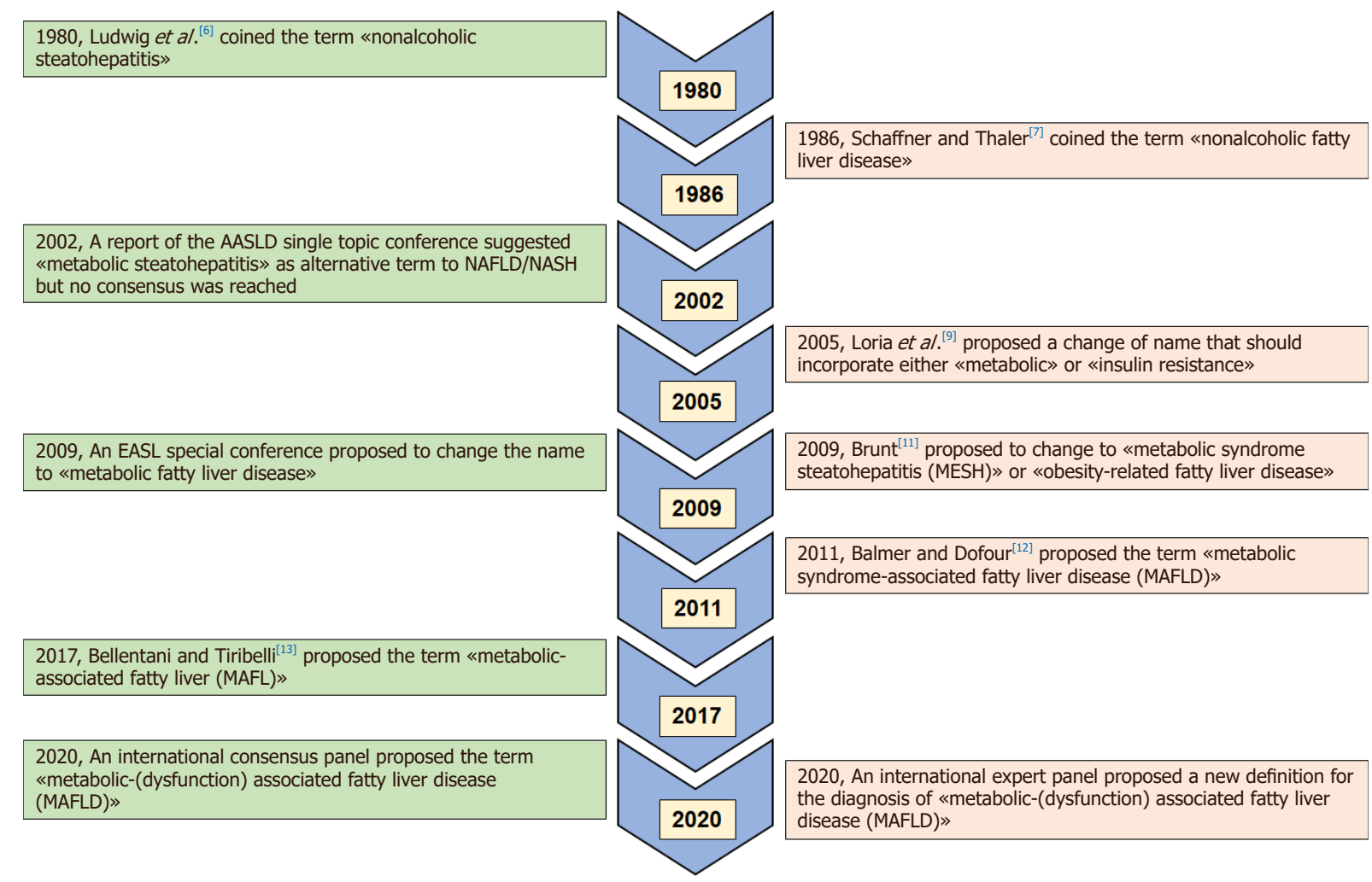

Figure 1. Schematic timeline of key suggestions for the revising the nomenclature of fatty liver disease from NAFLD to MAFLD ${ }^{[4-13]}$. NAFLD: nonalcoholic fatty liver disease; MAFLD: metabolic dysfunction-associated fatty liver disease

It is important to highlight that these "positive" diagnostic criteria recognize that MAFLD can also frequently co-exist with other conditions (e.g., significant alcohol consumption, viral hepatitis or other known chronic liver diseases), but the exclusion of these conditions is not a prerequisite for the diagnosis of MAFLD. Indeed, one of the advantages of the proposed diagnostic criteria for MAFLD is that they do not rely on exclusion of significant alcohol intake to establish the diagnosis. Individuals with MAFLD who also have one (or more) of these conditions should be defined as having dual (or more) aetiology of fatty liver disease ${ }^{[5]}$, and it is likely that these individuals will have a different natural history and response to different treatments. For instance, previous studies have shown that a significant proportion of the general adult population (e.g., up to nearly $1 \%$ of United States individuals from the National Health and Nutrition Examination Survey database 2003-2014) ${ }^{[20]}$ may be affected by both alcoholic disease and NAFLD (referred as BAFLD) and these patients tend to have more severe liver-related outcomes, given the synergistic interactions between alcohol consumption and features of the metabolic syndrome, such as obesity and type 2 diabetes mellitus ${ }^{[20,21]}$.

The rationale for the use of these "positive" diagnostic criteria for MAFLD largely stem from the fact that there is a strong pathophysiological link between this liver disease and the presence of underlying abnormalities in metabolic health (namely overweight/obesity, type 2 diabetes, insulin resistance or other metabolic risk abnormalities $)^{[17-19,21-29]}$. In addition, there is also convincing evidence that the coexistence of these metabolic risk abnormalities is not only one of the strongest risk factors of liver disease progression but also extra-hepatic clinical outcomes (mainly cardiovascular disease, chronic kidney disease and certain types of extra-hepatic cancers) in this patient population ${ }^{[17-19,23-31]}$. As depicted in Figure 2, the new diagnostic criteria for MAFLD would also be able to capture the whole phenotypical spectrum of fatty liver disease from that associated with metabolically unhealthy normal weight to metabolically unhealthy obesity. 


\section{Hepatic steatosis in adults}

(detected either by liver biopsy, imaging techniques or blood biomarkers/scores)

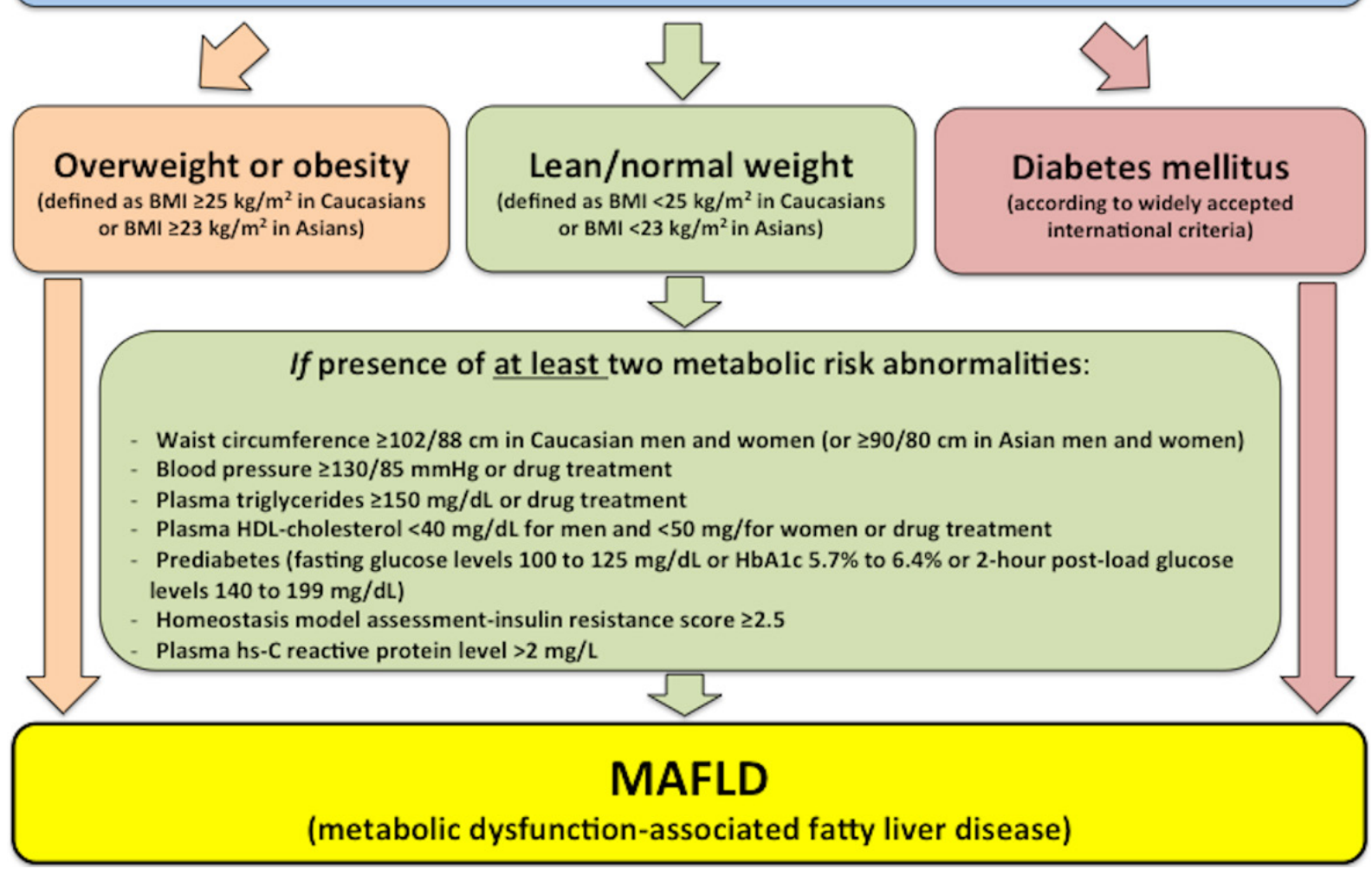

Figure 2. Flowchart for the proposed "positive" diagnostic criteria for metabolic dysfunction-associated fatty liver disease (MAFLD) in adult individuals. These diagnostic criteria are not applicable to the paediatric population. This figure is reproduced with permission from Eslam et al. ${ }^{[5]}$

The panel of international experts has also proposed that MAFLD should be the single "overarching" term to describe this fatty liver disease ${ }^{[4,5]}$. Similar to that accepted for other chronic liver diseases, they proposed that the histological severity of liver disease should be defined by its grade of activity and its stage of fibrosis ${ }^{[5]}$. This recognizes that the grade of MAFLD activity is a disease continuum and the all-embracing term of MAFLD should replace the current stratification of liver disease into NASH and non-NASH, which may also have some important limitations (e.g., there is significant inter-observer and intra-observer variability for the histologic confirmation of NASH, particularly for ballooned hepatocytes) ${ }^{[5]}$. From a clinical and pathological perspective, this suggestion should also result in improved case identification, while sub-classification of MAFLD (based on the use of liver histology) may also capture histological changes in disease status with relevant impacts on the disease course ${ }^{[5]}$. As suggested by the panel of international experts, it is reasonable to assume that MAFLD could be sub-classified in the foreseeable future, based on new epidemiological and experimental data that might suggest the predominant pathophysiological pathway that drives the development of specific features of liver histology (steatosis, necro-inflammation or fibrosis), but which might lead to different hepatic and extra-hepatic clinical complications (e.g., cardiovascular disease, extra-hepatic cancers or chronic kidney disease) ${ }^{[5]}$.

The expert panel has also proposed a set of diagnostic criteria to define MAFLD-related cirrhosis (in order to avoid the use of the term "cryptogenic cirrhosis" in this patient group) and proposed a conceptual framework to consider other causes of fatty liver disease ${ }^{[5]}$. The diagnostic criteria for MAFLD-related cirrhosis include patients with established cirrhosis in the absence of the typical histological signs 
suggestive of steatohepatitis, who meet at least one of the following criteria: past or present evidence of metabolic risk abnormalities that meet the criteria to diagnose MAFLD (as described in Figure 2) with at least one of the following diagnostic criteria, namely documentation of MAFLD on a previous liver biopsy, or historical documentation of hepatic steatosis by imaging methods ${ }^{[5]}$. Notably, a history of past alcohol intake should be considered as patients may have a dual disease aetiology with alcohol use disorder ${ }^{[5]}$.

Finally, the panel of international experts has also proposed the new definition of MAFLD in order to bring more clarity to the distinction between the diagnostic criteria used in clinical practice to define this common liver disease and the inclusion criteria used for future research studies or randomized controlled trials $^{[5]}$. However, these considerations in no way detract the conduct from ongoing trials, nor does it affect the diagnostic criteria proposed for MAFLD. As also suggested by the panel of international experts, it is reasonable to hypothesize that the use of the newly proposed "positive" criteria for the diagnosis of MAFLD and the exclusion of individuals with hepatic steatosis who do not have coexisting metabolic dysfunction will render study cohorts less heterogeneous, thereby increasing the probability of detecting a positive effect of clinical approaches targeting MAFLD ${ }^{[5]}$.

\section{MAFLD and NAFLD: are two terms interchangeable?}

It is possible that a consequence of implementing the proposed change in terminology will also highlight some new categories of fatty liver disease. This might also foster new discoveries of the causes, mechanisms, classification and treatment of fatty liver disease ${ }^{[5]}$.

In line with this hypothesis, Lin et al. ${ }^{[32]}$ have recently compared the characteristics of MAFLD and NAFLD in a nationally representative cohort of nearly 13,000 United States adults with liver ultrasonography and complete laboratory data. Interestingly, using the National Health and Nutrition Examination Survey (NHANES-III) 1988-1994 database, the authors reported that NAFLD was present in $33.2 \%$ of participants, while MAFLD was present in $31.2 \%$. In addition, compared to those with NAFLD, patients with MAFLD were more likely to have multiple metabolic comorbidities (e.g., obesity, hypertension and type 2 diabetes) and more cases with advanced liver fibrosis as detected by non-invasive fibrosis scores ${ }^{[32]}$. These findings suggest that MAFLD definition is more accurate than NAFLD definition in identifying those patients with hepatic steatosis who are at higher risk of disease progression or have a greater risk of cardiovascular disease.

As depicted in Figure 3, these "real-world" data clearly indicate that there is an excellent concordance between MAFLD and NAFLD (with a calculated Cohen's kappa coefficient of 0.92) ${ }^{[33]}$. However, NAFLD and MAFLD definitions do not identify exactly the same individuals and, therefore, the two terms are not fully interchangeable. Looking at these "real-world" data from adult individuals in the United States, it is evident that not all individuals with NAFLD have MAFLD and vice versa. Indeed, as shown in Figure 3, there are some individuals with MAFLD and coexisting liver diseases who do not have NAFLD. There is also a non-negligible proportion of individuals (620/4,347; nearly 15\%) previously deemed as having NAFLD who do not have MAFLD, and that cannot be attributed to significant alcohol consumption (based on self-reported history that might also be under-reported in clinical practice) or other known causes of liver disease ${ }^{[32]}$. Notably, as reported in the study by Lin et al. ${ }^{[32]}$, these latter individuals are predominantly of female sex ( 60\% women), young (mean \pm SD: $35 \pm 13.4$ years) and have "lean" NAFLD (mean body mass index: $21.7 \pm 2.1 \mathrm{~kg} / \mathrm{m}^{2}$ ) without coexisting metabolic dysregulation, as defined by the diagnostic criteria of MAFLD. Most importantly, these individuals also have a (relatively) low proportion of advanced liver fibrosis ${ }^{[32]}$. Unfortunately, no information is available about patatin-like phospholipase domaincontaining protein-3 (PNPLA3) I148M variant, trans-membrane-6 superfamily member 2 (TM6SF2) E167K variant or other NAFLD-related genetic variants in these "lean" NAFLD individuals without metabolic dysregulation. 


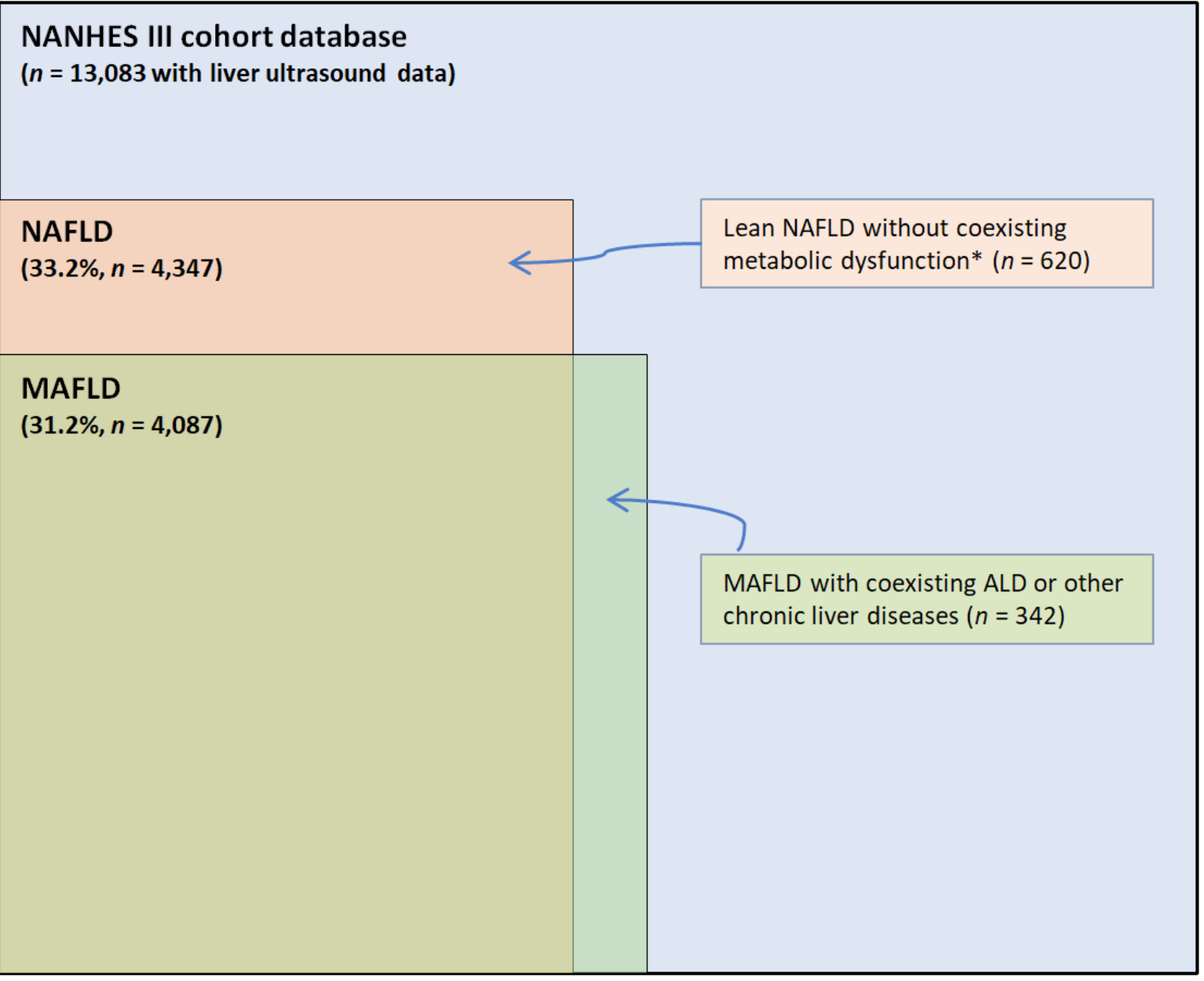

Figure 3. Concordance between MAFLD and NAFLD diagnostic criteria in the Third National Health and Nutrition Examination Survey (NHANES) 1988-1994 database. Data are extrapolated from the study by Lin et al. ${ }^{[32]}$. This figure is quoted with permission from Targher G. ${ }^{[33]}$. *Metabolic dysfunction was defined according to MAFLD diagnostic criteria. MAFLD: metabolic dysfunction-associated fatty liver disease; NAFLD: nonalcoholic fatty liver disease; ALD: alcoholic liver disease

That said, further large cohort studies are needed to validate and corroborate the aforementioned findings in European, American and Asian populations (where the overall prevalence of "lean" NAFLD is greater than in United States) ${ }^{[34]}$ and, most importantly, to examine the risk of developing adverse liver-related and extra-hepatic outcomes (e.g., cardiovascular disease, chronic kidney disease and extra-hepatic cancers) in patients with MAFLD compared with those with NAFLD. In addition, further research is also needed to better understand the risk of disease progression amongst "lean" NAFLD individuals without any metabolic dysregulation.

\section{CHALLENGES AND THE WAY FORWARD}

In addition to the challenges for the MAFLD field that have been outlined in the two new position papers (e.g., definition of "metabolic health", different disease subtypes) ${ }^{[4,5]}$, words of caution have also been expressed by a distinguished group of United States experts regarding the possible implications of a premature change in NAFLD terminology ${ }^{[35]}$. In particular, although these colleagues are in agreement that the new term MAFLD reflects more accurately the relevant metabolic risk factors than the age-old term NAFLD, they also suggest that a change in terminology for a disease entity is only justified when there is a better understanding of its pathogenesis, risk stratification and molecular phenotyping ${ }^{[35]}$. That said, one 
good reason to change the nomenclature and the diagnostic criteria for defining this fatty liver disease is that current diagnostic criteria focused on diagnosing NASH by histology only is hindering both the testing of novel treatments for NAFLD and the discovery of new biomarkers. As the change in nomenclature and diagnostic criteria for this fatty liver disease have not been accepted by the major scientific societies, it has been recommended that an international consensus conference should be jointly organized by the major relevant scientific societies along with the patient advocacy organizations, biopharmaceutical industry, regulatory agencies and policy makers to thoughtfully consider any implications of a change in terminology of NAFLD as we know it ${ }^{[35]}$. Following the same process that occurred for changing the term "primary biliary cirrhosis" to "primary biliary cholangitis", all the main scientific societies should approve the different steps, reach a consensus and publish a position paper on MAFLD.

\section{CONCLUSION}

Based on pathophysiological insights gained from the past decades, two new articles from a panel of international experts from 22 countries have proposed a new name and definition for NAFLD in adults - i.e., MAFLD $^{[4,5]}$. We believe that a change in the nomenclature and definition for this burdensome liver disease affecting nearly one billion people globally is overdue, as knowledge gained from the last two decades has clearly established that MAFLD is a (predominantly) metabolic liver disease. Furthermore, the term "nonalcoholic" over-emphasizes the absence of significant alcohol consumption and does not acknowledge the importance of overweight/obesity, type 2 diabetes mellitus, insulin resistance and other metabolic risk abnormalities that fuel the risk of liver disease progression and the development of serious adverse extrahepatic outcomes (e.g., cardiovascular disease, extra-hepatic malignancies or chronic kidney disease $)^{[36-41]}$. To date, it is known that there is a substantial under-appreciation of NAFLD by both primary care clinicians and patients ${ }^{[42,43]}$. We believe that the proposed change of the name from NAFLD to MAFLD holds promise to aid in increasing awareness of this liver disease and decreasing its possible social stigma due to its link to alcohol intake. It is reasonable to propose that this new definition might also promote the establishment of MAFLD clinics run jointly by hepatologists and diabetologists to further improve patient care. To date, however, a number of challenges and uncertainties remain before making this stepchange. As discussed above, a change to the nomenclature and diagnostic criteria for NAFLD is a potential first relevant step to acknowledging that NAFLD is an important metabolic liver disease whose aetiology and pathogenesis extend beyond the liver. Indeed, it is known that NAFLD is a novel risk factor for the development of cardiovascular disease, chronic kidney disease, type 2 diabetes and some extra-hepatic malignancies (e.g., colorectal cancers) ${ }^{[36-41]}$. In the meantime, an international consensus conference should be jointly organized by the major relevant scientific societies to discuss the implications of a change in terminology from NAFLD to MAFLD and to achieve a consensus regarding any change in nomenclature and definition for this common metabolic liver disease.

\section{DECLARATIONS}

\section{Authors' contributions}

Made substantial contributions to conception and design of the study and contributed equally to write this commentary article: Targher G, Byrne CD

\section{Availability of data and materials}

Not applicable.

\section{Financial support and sponsorship}

None.

\section{Conflicts of interest}

Both authors declared that there are no conflicts of interest. Targher G was a coauthor of one of the two recent articles ${ }^{[5]}$ proposing the change of terminology from NAFLD to MAFLD. 


\section{Ethical approval and consent to participate}

Not applicable.

\section{Consent for publication}

Not applicable.

\section{Copyright}

(c) The Author(s) 2020.

\section{REFERENCES}

1. Bellentani S. The epidemiology of non-alcoholic fatty liver disease. Liver Int 2017;37 Suppl 1:81-4.

2. Younossi Z, Anstee QM, Marietti M, Hardy T, Henry L, et al. Global burden of NAFLD and NASH: trends, predictions, risk factors and prevention. Nat Rev Gastroenterol Hepatol 2018;15:11-20.

3. Byrne CD, Targher G. NAFLD: a multisystem disease. J Hepatol 2015;62:S47-64.

4. Eslam M, Sanyal AJ, George J; International Consensus Panel. MAFLD: a consensus-driven proposed nomenclature for metabolic associated fatty liver disease. Gastroenterology 2020;158:1999-2014.e1.

5. Eslam M, Newsome PN, Sarin SK, Anstee QM, Targher G, et al. A new definition for metabolic dysfunction-associated fatty liver disease: an international expert consensus statement. J Hepatol 2020;73:202-9.

6. Ludwig J, Viggiano TR, McGill DB, Oh BJ. Nonalcoholic steatohepatitis: mayo clinic experiences with a hitherto unnamed disease. Mayo Clin Proc 1980;55:434-8.

7. Schaffner F, Thaler H. Nonalcoholic fatty liver disease. Prog Liver Dis 1986;8:283-98.

8. Neuschwander-Tetri BA, Caldwell SH. Nonalcoholic steatohepatitis: summary of an AASLD single topic conference. Hepatology 2003;37:1202-19.

9. Loria P, Lonardo A, Carulli N. Should nonalcoholic fatty liver disease be renamed? Dig Dis 2005;23:72-82.

10. Ratziu V, Bellentani S, Cortez-Pinto H, Day C, Marchesini G. A position statement on NAFLD/NASH based on the EASL 2009 special conference. J Hepatol 2010;53:372-84.

11. Brunt EM. Histopathology of non-alcoholic fatty liver disease. Clin Liver Dis 2009;13:533-44.

12. Balmer ML, Dufour JF. Non-alcoholic steatohepatitis - from NAFLD to MAFLD. Ther Umsch 2011;68:183-8.

13. Bellentani S, Tiribelli C. Is it time to change NAFLD and NASH nomenclature? Lancet Gastroenterol Hepatol 2017;2:547-8.

14. Valenti L, Pelusi S. Redefining fatty liver disease classification in 2020. Liver Int 2020;40:1016-7.

15. Fouad Y, Waked I, Bollipo S, Gomaa A, Ajlouni Y, et al. What's in a name? Renaming 'NAFLD' to 'MAFLD'. Liver Int 2020;40:1254-61.

16. Tilg H, Effenberger M. From NAFLD to MAFLD: when pathophysiology succeeds. Nat Rev Gastroenterol Hepatol 2020;17:387-8.

17. European Association for the Study of the Liver (EASL), European Association for the Study of Diabetes (EASD), European Association for the Study of Obesity (EASO). EASL-EASD-EASO Clinical Practice Guidelines for the management of non-alcoholic fatty liver disease. J Hepatol 2016;64:1388-402.

18. Italian Association for the Study of the Liver (AISF). AISF position paper on nonalcoholic fatty liver disease (NAFLD): updates and future directions. Dig Liver Dis 2017;49:471-83.

19. Chalasani N, Younossi Z, Lavine JE, Charlton M, Cusi K, et al. The diagnosis and management of nonalcoholic fatty liver disease: practice guidance from the American association for the study of liver diseases. Hepatology 2018;67:328-57.

20. Khoudari G, Singh A, Noureddin M, Fritze D, Lopez R, et al. Characterization of patients with both alcoholic and nonalcoholic fatty liver disease in a large United States cohort. World J Hepatol 2019;11:710-8.

21. Åberg F, Helenius-Hietala J, Puukka P, Färkkilä M, Jula A. Interaction between alcohol consumption and metabolic syndrome in predicting severe liver disease in the general population. Hepatology 2018;67:2141-9.

22. Alberti KG, Eckel RH, Grundy SM, Zimmet PZ, Cleeman JI, et al; International Diabetes Federation Task Force on Epidemiology and Prevention, Hational Heart, Lung, and Blood Institute, American Heart Association, World Heart Federation, International Atherosclerosis Society, International Association for the Study of Obesity. Harmonizing the metabolic syndrome: a joint interim statement of the International Diabetes Federation Task Force on Epidemiology and Prevention; National Heart, Lung, and Blood Institute; American Heart Association; World Heart Federation; International Atherosclerosis Society; and International Association for the Study of Obesity. Circulation 2009;120:1640-5.

23. Bugianesi E, Zannoni C, Vanni E, Marzocchi R, Marchesini G. Non-alcoholic fatty liver and insulin resistance: a cause-effect relationship? Dig Liver Dis 2004;36:165-73.

24. Marchesini G, Marzocchi R. Metabolic syndrome and NASH. Clin Liver Dis 2007;11:105-17, ix.

25. Anstee QM, Targher G, Day CP. Progression of NAFLD to diabetes mellitus, cardiovascular disease or cirrhosis. Nat Rev Gastroenterol Hepatol 2013;10:330-44.

26. Lonardo A, Ballestri S, Marchesini G, Angulo P, Loria P. Nonalcoholic fatty liver disease: a precursor of the metabolic syndrome. Dig Liver Dis 2015;47:181-90.

27. Lonardo A, Bellentani S, Argo CK, Ballestri S, Byrne CD, et al; Non-alcoholic Fatty Liver Disease Study Group. Epidemiological modifiers of non-alcoholic fatty liver disease: focus on high-risk groups. Dig Liver Dis 2015;47:997-1006. 
28. Tilg H, Moschen AR, Roden M. NAFLD and diabetes mellitus. Nat Rev Gastroenterol Hepatol 2017;14:32-42.

29. Byrne CD, Patel J, Scorletti E, Targher G. Tests for diagnosing and monitoring non-alcoholic fatty liver disease in adults. BMJ 2018;362:k2734.

30. Gastaldelli A, Cusi K. From NASH to diabetes and from diabetes to NASH: Mechanisms and treatment options. JHEP Rep 2019;1:312-28.

31. Khan RS, Bril F, Cusi K, Newsome PN. Modulation of insulin resistance in nonalcoholic fatty liver disease. Hepatology 2019;70:711-24.

32. Lin S, Huang J, Wang M, Kumar R, Liu Y, et al. Comparison of MAFLD and NAFLD diagnostic criteria in real world. Liver Int 2020:2082-9.

33. Targher G. Concordance of MAFLD and NAFLD diagnostic criteria in "real-world" data. Liver Int 2020; doi: 10.1111/liv.14623.

34. Lu FB, Zheng KI, Rios RS, Targher G, Byrne CD, et al. Global epidemiology of lean non-alcoholic fatty liver disease: a systematic review and meta-analysis. J Gastroenterol Hepatol 2020; doi: 10.1111/jgh.15156.

35. Younossi ZM, Rinella ME, Sanyal A, Harrison SA, Brunt E, et al. From NAFLD to MAFLD: implications of a premature change in terminology. Hepatology 2020; doi: 10.1002/hep.31420.

36. Yki-järvinen H. Non-alcoholic fatty liver disease as a cause and a consequence of metabolic syndrome. Lancet Diabetes Endocrinol 2014;2:901-10.

37. Adams LA, Anstee QM, Tilg H, Targher G. Non-alcoholic fatty liver disease and its relationship with cardiovascular disease and other extrahepatic diseases. Gut 2017;66:1138-53.

38. Stefan N, Häring H, Cusi K. Non-alcoholic fatty liver disease: causes, diagnosis, cardiometabolic consequences, and treatment strategies. Lancet Diabetes Endocrinol 2019;7:313-24.

39. Targher G, Byrne CD, Tilg H. NAFLD and increased risk of cardiovascular disease: clinical associations, pathophysiological mechanisms and pharmacological implications. Gut 2020;69:1691-705.

40. Byrne CD, Targher G. NAFLD as a driver of chronic kidney disease. J Hepatol 2020;72:785-801.

41. Mantovani A, Scorletti E, Mosca A, Alisi A, Byrne CD, et al. Complications, morbidity and mortality of nonalcoholic fatty liver disease. Metabolism 2020:154170.

42. Wieland AC, Mettler P, McDermott MT, Crane LA, Cicutto LC, et al. Low awareness of nonalcoholic fatty liver disease among patients at high metabolic risk. J Clin Gastroenterol 2015;49:e6-10.

43. Alemany-Pagès M, Moura-Ramos M, Araújo S, Macedo MP, Ribeiro RT, et al. Insights from qualitative research on NAFLD awareness with a cohort of T2DM patients: time to go public with insulin resistance? BMC Public Health 2020;20:1142. 